\title{
Article \\ Optical Performance and Moisture Stability Enhancement of Flexible Luminescent Films Based on Quantum-Dot/Epoxy Composite Particles
}

\author{
Guanwei Liang ${ }^{1}$, Yong Tang ${ }^{1}$, Jiarui Huang ${ }^{1}$, Jiasheng $\mathrm{Li}^{1}$, Yikai Yuan ${ }^{2}$, Shu Yang ${ }^{1}$ and Zongtao $\mathrm{Li}^{1,2, *(\mathbb{D})}$ \\ 1 National and Local Joint Engineering Research Center of Semiconductor Display and VLC Devices, \\ South China University of Technology, Guangzhou 510640, China; megwliang@yeah.net (G.L.); \\ ytang@scut.edu.cn (Y.T.); 202021002916@mail.scut.edu.cn (J.H.); Jiasli@foxmail.com (J.L.); \\ yangshme@scut.edu.cn (S.Y.) \\ 2 Guangdong Provincial Key Laboratory of Semiconductor Micro Display, Foshan Nationstar Optoelectronics \\ Company Ltd., Foshan 528000, China; yuanyikai@nationstar.com \\ * Correspondence: meztli@scut.edu.cn
}

check for updates

Citation: Liang, G.; Tang, Y.; Huang, J.; Li, J.; Yuan, Y.; Yang, S.; Li, Z. Optical Performance and Moisture Stability Enhancement of Flexible Luminescent Films Based on Quantum-Dot/Epoxy Composite Particles. Nanomaterials 2021, 11, 2100. https://doi.org/10.3390/ nano11082100

Academic Editor: Mohammad Jawaid

Received: 8 July 2021

Accepted: 31 July 2021

Published: 18 August 2021

Publisher's Note: MDPI stays neutral with regard to jurisdictional claims in published maps and institutional affiliations.

Copyright: (c) 2021 by the authors. Licensee MDPI, Basel, Switzerland. This article is an open access article distributed under the terms and conditions of the Creative Commons Attribution (CC BY) license (https:/ / creativecommons.org/licenses/by/ $4.0 /)$.

\begin{abstract}
Quantum dots (QDs) have been widely applied in luminescent sources due to their strong optical characteristics. However, a moisture environment causes their quenching, leading to an inferior optical performance in commercial applications. In this study, based on the high moisture resistance of epoxy resin, a novel epoxy/QDs composite particle structure was proposed to solve this issue. Flexible luminescent films could be obtained by packaging composite particles in silicone resin, combining the hydrophobicity of epoxy resin and the flexibility of PDMS simultaneously. The photoluminescence and light extraction were improved due to the scattering properties of the structure of composite particles, which was caused by the refractive index mismatch between the epoxy and silicone resin. Compared to the QD/silicone film under similar lighting conditions, the proposed flexible film demonstrated increased light efficiency as well as high moisture stability. The results revealed that a light-emitting diode (LED) device using the composite particle flexible (CPF) structure obtained a $34.2 \%$ performance enhancement in luminous efficiency as well as a $32 \%$ improvement in color conversion efficiency compared to those of devices with QD/silicone film (QSF) structure. Furthermore, the CPF structure exhibited strong thermal and moisture stability against extreme ambient conditions of $85{ }^{\circ} \mathrm{C}$ and $85 \%$ relative humidity simultaneously. The normalized luminous flux degradation of devices embedded in CPF and QSF structures after aging for $118 \mathrm{~h}$ were $\sim 20.2 \%$ and $\sim 43.8 \%$, respectively. The satisfactory performance of the CPF structure in terms of optical and moisture stability shows its great potential value in flexible commercial QD-based LED displays and lighting applications.
\end{abstract}

Keywords: quantum dots; composite particles; luminous efficiency; moisture stability

\section{Introduction}

Quantum dots (QDs) have been attracting great attention and have gradually become one of the most promising color-conversion materials in white light-emitting diodes (LEDs), because of their high quantum yield, high color rendering quality, and narrow emission angle [1-6]. They are widely used in LED encapsulation as photoluminescence materials for next-generation lighting and display devices [7-9]. Various breakthroughs regarding the luminous efficacy of QD-based LEDs have been achieved gradually, and the efficacy of QD-based LEDs has reached 200 lumens per watt [10]. In QD-based LED encapsulation, QDs are dissolved in polymer when fabricating composite materials to prevent them from moisture and oxidation [11]. Meanwhile, silicone is the most common packaging material because of its excellent flexibility, mechanical strength, optical transmittance, as well as thermal stability [12-14]; however, it suffers from bad moisture stability. The moisture 
and/or oxygen may lead to the aggregation, surface destruction, and photoluminescence quenching of QDs and thus, negatively affect the lifetime of QD-based LED devices [15,16]. To date, numerous packaging methods have been proposed to solve these issues, such as chemically passivating the QD surface during the chemical synthesis process $[17,18]$. Using polymer materials to protect the QDs is the most common QD encapsulation approach. For instance, CdS/PMMA core/shell nanoparticles are fabricated to maintain the optical properties of QDs while effectively protecting them from environmental perturbation [19]. Recently, a super-hydrophobic microstructure was proposed to enhance the water stability of QDs. Xuan et al., embedded QDs into a super-hydrophobic porous organic polymer framework [20]. QDs have been also incorporated into a porous matrix to isolate moisture [21,22]. Moreover, superhydrophobic nanosilica particles have been coated on the QDs/polymer films to prevent moisture from penetrating the films [23]. $\mathrm{QD} /$ polymer fibers prepared by electrospinning demonstrate a new and effective way to enhance QD moisture stability, which can be used to prepare water-resistant hybrid membranes [24,25]. Previous studies have indicated that QD encapsulation by a polymer matrix is the most efficient approach to enhance QD moisture stability. However, the abovementioned methods may involve complex material selection and fabrication processes, which may negatively affect the optical properties of QDs. Furthermore, with regard to QD-LED packaging, limited studies have focused on device moisture stability, which has been mostly ignored to consider the optical performance. The addition of anti-moisture materials or structure could decrease the device's lighting efficiency [23]. The main challenge is to realize a QD/polymer composite matrix that simultaneously exhibits light efficiency and moisture stability.

Epoxy resin is usually used in outdoor LED encapsulation owing to its high optical stability and moisture resistance [26,27], and always as the encapsulation resin for QDs [28]. However, the curing epoxy demonstrates a high degree of hardness, which limits its application in devices requiring flexibility, such as wearable devices. Nevertheless, the epoxy can be ground into micro particles to realize a QD/epoxy composite matrix, which can be easily dissolved in traditional packaging materials such as silicone resin. In addition, the diameter of QD is about several to a hundred nanometers [29], and its weak scattering ability may be compensated by the micro particles [30]. The existence of difference indices between the packaging materials is beneficial to enhance the light scattering [7,31], which contributes to improving the QD photoluminescence. Based on the fabrication of a QD/epoxy composite structure, the light path can be controlled by packaging the composite materials in the silicone resins. The composite particle structure can be an alternative method to enhance both the device's moisture stability and light efficiency.

In this study, QDs and epoxy resin composite particles were fabricated to enhance the optical performance and moisture stability of flexible luminescent films. The fabrication and optical behaviors of the composite particle structure were thoroughly investigated. Finally, flexible QD films and QD-based LEDs with similar lighting effects were prepared and tested to confirm the effect of the composite particles. Compared to the traditional packaging structure obtained by dissolving QDs in silicone resin, the results obtained for the composite structure in this study illustrated that LED devices with the composite particle film $(\mathrm{CPF})$ can greatly enhance the luminous and color-conversion efficiencies as well as moisture stability. Therefore, the CPF structure can be an effective and suitable candidate for QD powders for manufacturing flexible LED devices in the industry.

\section{Experimental and Measurement}

\subsection{Composite Particles Preparation}

In this study, CdSe/ZnS core-shell QDs with an emission peak of $570 \mathrm{~nm}$ were bought from Beida Jubang Co. Ltd (Beijing, China). as in our previous works [29], which quantum yield is about $85 \%$. First, $120 \mathrm{mg}$ of QDs was dissolved in chloroform, followed by the addition of $4 \mathrm{~g}$ of epoxy resin (IK0010, IK INABATA \& Co., Ltd., Tokyo, Japan) into the mixture. Subsequently, in order to completely volatile the chloroform, the mixture was 
subjected to centrifugal stirring with $400 \mathrm{rpm}$ revolution rate and $300 \mathrm{rpm}$ rotation rate for $2 \mathrm{~h}$ using a mixer (FY-821, Shenzhen Fuyuanxiang Electronic Equipment Co., Ltd., Shenzhen, China). Subsequently, $4 \mathrm{~g}$ of epoxy crossing resin was added to the mixture, and the mixture was stirred in vacuum to ensure uniform mixing and removal of bubbles. Finally, the mixture was tape-casted on a PET film and cured at $150{ }^{\circ} \mathrm{C}$ for $3 \mathrm{~h}$. The curing films were cut into small pieces and then ground at $450 \mathrm{rpm}$ for $5 \mathrm{~h}$ by a ball grinder. This process is illustrated in detail in Figure 1. After filtering, uniform QDs/epoxy composite particles (QE CPs) were obtained; the photograph of the composite particles is shown in Figure 1d.

(a)

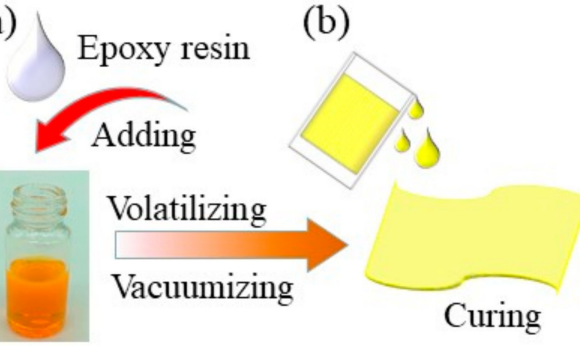

(b)
QD in chloroform

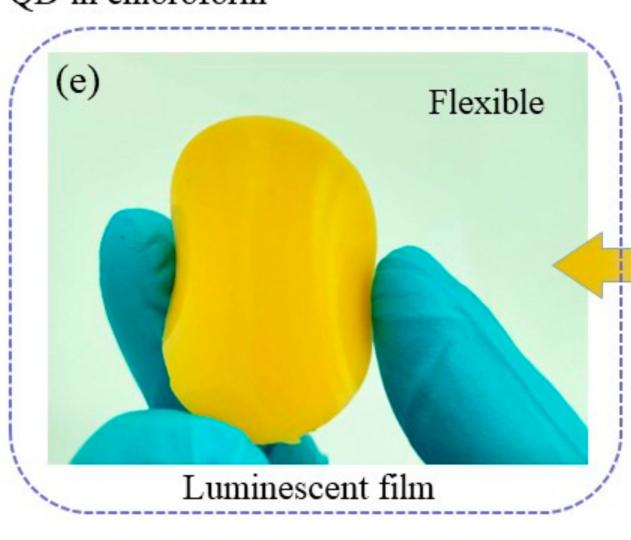

Cutting

Grinding

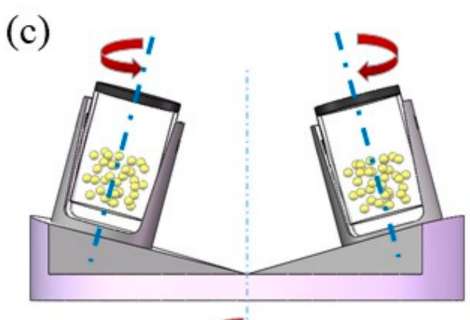

Filtering

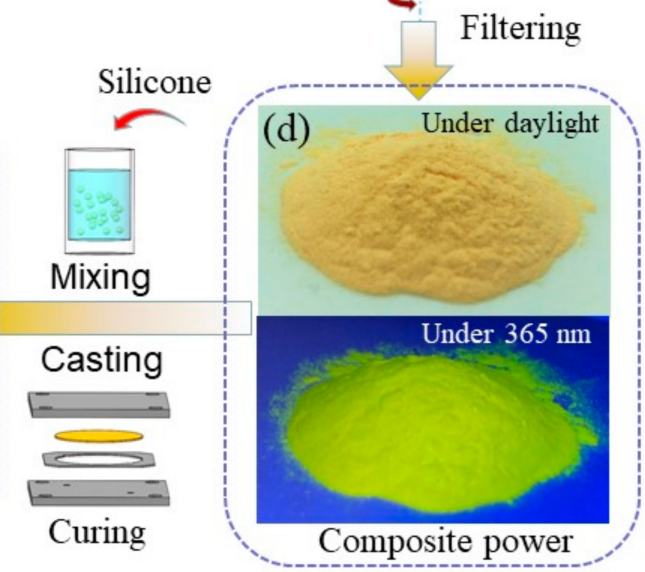

Figure 1. (a-c) Fabrication process of QD/epoxy composite particles. (d) Images of composite particles under daylight and $365 \mathrm{~nm}$. (e) Images of flexible luminescent film fabricated by mold casting method.

\subsection{Fabrication of Flexible Luminescent Films}

To obtain a flexible film, the composite particles were packaged in the polydimethylsiloxane (PDMS, Dow Corning Sylgard-184, Midland, MI, USA) silicone resin. It can easily achieve different lighting effects by varying the composite particle concentration from 0.09 to $0.37 \mathrm{wt} \%$. The QD concentration is defined as the ratio of QD mass to the mass of resin. After uniformly mixing the composite particles and PDMS, the mixture was cast and cured at $110^{\circ} \mathrm{C}$ for $0.5 \mathrm{~h}$ using a mold casting method. Under the same curing condition, pure PDMS and epoxy films were fabricated by a standard mold to study their mechanical properties. A photograph of the prepared composite particle flexible (CPF) films is shown in Figure 1e. For comparison, a QD/silicone (QS) film was also fabricated by directly dissolving QDs in pure PDMS silicone. The QD concentration of QS films was adjusted to achieve the same lighting effect with the CPF-structured films.

\subsection{Characterizations and Measurements}

The morphology of composite particles was characterized by field emission scanning electron microscopy (FE-SEM, Zeiss ${ }^{\circledR}$ Merlin, Oberkohen, Germany). The diameter distribution of composite particles was measured using Image J software. Elemental mapping images of the composite particles were also analyzed by Zeiss SmartEDX (Oxford, UK) to study the element mass ratio distribution on the surface of composite particles. The 
high-resolution transmission electron microscope (HRTEM) images of CdSe/ZnS core-shell QDs in epoxy resin were measured by a field emission transmission electron microscopy (JEM-1400 PLUS, JEOL, Tokyo, Japan). The TEM samples were sectioned by an ultramicrotome (UC7/FC7, LEICA, Wetzlar, Germany) at room temperature, and used for measurement. The FTIR spectra were tested by a Fourier Transform infrared spectroscopy (Nicolet IS50, Thermo Fisher Scientific Inc., MA, USA). The stress-strain curves of the PDMS and epoxy films were obtained through testing with a tensile testing machine (WDW-01A, Jinan Fine Experimental Instrument Co., Ltd., Jinan, China). During the tensile test, the stretching speed was maintained at $2 \mathrm{~mm} / \mathrm{min}$. The diffuse transmittance and absorbance were measured using a dual-beam UV-Vis spectrophotometer (TU-1901, Beijing Persee General Instrument Co., Ltd., Beijing, China). Details of the measurement of asymmetric bidirectional transmittance distribution function (BTDF) can be found in our previous report [32], which evaluates the scattering ability of the films. The photoluminescence (PL) was tested by a fluorescence spectrophotometer (RF-6000, Shimadzu, Japan) with an excitation wavelength at $365 \mathrm{~nm}$. The optical performance of the LED devices with QD films was tested on an integrating sphere system including a 0.5 -m-diameter integrating sphere, a spectrometer (Otsuka LE5400, Otsuka Electronics Co., Ltd., Osaka, Japan), and a DC power supply (Keithley 2425, Tektronix, Inc., Beaverton, USA). Blue COB-LEDs with sixteen chips were used to excite the remote QD films for white lights. The optical performance of two types of structural films was tested under different driving currents varying from 50 to $1000 \mathrm{~mA}$. For the aging process, the CPF and QS films were placed in a thermostatic and humidistatic machine with ambient conditions of $85{ }^{\circ} \mathrm{C}$ and $85 \%$ relative humidity. After aging for different times, two types of structural films were coated on the COB devices to investigate their optical performance.

\section{Results and Discussions}

To study the morphology of the composite particles, Figure 2a shows the SEM image of the composite particles after grinding. It can be observed that the composite particles with spherical morphology exhibit a good dispersion and present a uniform diameter distribution. A total of 250 pieces of composite particles were used to calculate for average diameters; the results are shown in Figure 2c. The composite particles exhibit a concentrated distribution with an average diameter of about $40 \mu \mathrm{m}$.

As shown in Figure 1d, the composite particles show bright yellow emission under $365 \mathrm{~nm}$, indicating that an effective package of QDs and epoxy composite particles was built. The QD/epoxy composite matrix can be ground into particles easily. To characterize the QD distribution in the epoxy, the energy dispersive spectroscopy (EDS) of composite particles was performed; the results are shown in Figure $2 b, d$,e. From Figure $2 b$, it is seen that the composite particles produce a rough surface after grinding, which may cause a change in incident light transfer direction. The element content measurement results (Figure 2d) show that a small amount of QD element content was detected, indicating that $\mathrm{CdSe} / \mathrm{ZnS}$ QDs seldom distribute on the epoxy surface. It can be inferred from the EDS mapping results that QDs are distributed in the inset of epoxy resin, which contributes to protecting QDs from water and oxygen. To further support this issue, Figure 2e shows the HRTEM images of QDs in epoxy resin. The measured QDs demonstrate uniform size distribution and QD are relatively evenly distributed in epoxy resin. There is no obvious QD aggregation in epoxy resin. Figure $2 f$ shows the FTIR spectra of the QDs and composite particles. Both the QDs and composite particles commonly exhibit the bands at 2846 and $2920 \mathrm{~cm}^{-1}$, which are attributed to the presence of $-\mathrm{CH}_{3}$ and $-\mathrm{CH}_{2}$ groups from the QD aliphatic surface light and species [17]. Typically, the composite particles exhibited a strong band at $1736 \mathrm{~cm}^{-1}$ from the $\mathrm{C}=\mathrm{O}$ stretching of the ester group [33]. It is also indicated that the QDs are distributed in the epoxy resin. The epoxy film exhibits a good moisture ability but strong rigidity, which is not desirable to fabricate luminescent films in flexible lighting and display devices. It can be seen from Figure $2 \mathrm{~g}$ that PDMS film demonstrates great flexibility. The addition of QDs slightly influenced the flexibility of CPF films, as 
shown in the inset of Figure 2g. To further study the mechanical properties of PDMS and epoxy films, a tensile test was carried out; the process is shown in Figure $2 \mathrm{~h}$. Figure $2 \mathrm{i}$ illustrates the representative stress-strain curve. Significantly, the PDMS film performs elastic deformation against stress while the epoxy film exhibits a higher elastic modulus compared to that of the PDMS film. During the elastic deformation stage, based on the proportional relationship between the stress and strain, the elastic modulus can be easily calculated from the stress-strain curve. The calculated elastic modulus of PDMS was about 1.57 MPa, while the CPF films exhibited a similar value. However, the epoxy film exhibited strong rigidity with a large elastic modulus at about $376.15 \mathrm{MPa}$. During these procedures, the combination of the respective advantage of PDMS and epoxy resin is essential to be used in flexible devices.
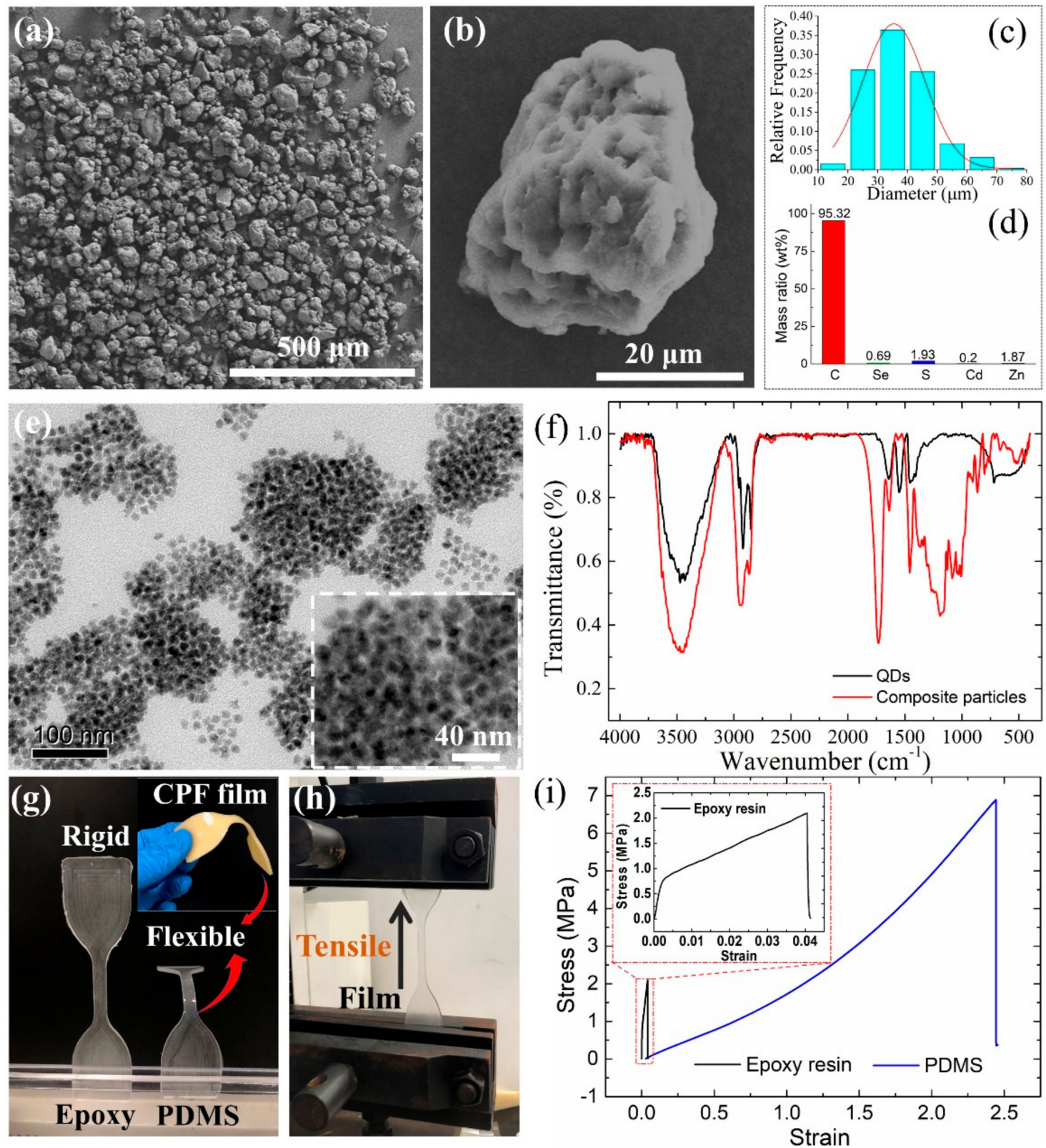

Figure 2. (a) SEM of composite particles. (b) Element mapping images of the composite particle. (c) Diameter distribution. (d) Element mass ratio distribution of corresponding mapping particle. (e) HRTEM images of QDs in epoxy resin. (f) FTIR spectra of QDs and composite particles. (g) Flexibility of PDMS film and rigidity of epoxy film. The inset shows the flexibility exhibition of CPF film. (h) Stress-strain curves measurement process. (i) Stress-strain curves of PDMS and epoxy films obtained from tensile test. 
To obtain film flexibility, the composite particles were encapsulated in silicone resin, and different composite particle concentration would change the film optical performance. The CPF films with different composite particle concentration were prepared to study these issues (Figure 3). The diffuse transmittance of CPF films related to composite particle concentration is illustrated in Figure 3a. As the QD concentration increased from 0.09 to $0.37 \mathrm{wt} \%$, the diffuse transmittance decreased gradually. The CPF films exhibited lower transmittance at shorter wavelengths and higher transmittance at longer wavelengths due to the light absorbance of QDs. Particularly, the diffuse transmittance at $450 \mathrm{~nm}$ decreased from $53 \%$ to $13 \%$, indicating a strong QD absorbance. Meanwhile, the addition of composite particles may change the light scattering performance caused by the index difference between the PDMS and epoxy resin. The BTDF normalized intensity was tested to evaluate the film scattering ability; the results are shown in Figure 3b. The normalized angular intensity distribution of the film was tested after passing a 632-nm laser beam through the testing films. There exists a significant peak at $0^{\circ}$ in pure PDMS as well as CPF films with a relatively low QD concentration of $0.09 \mathrm{wt} \%$. As the composite particle concentration increased, more light scatters to larger angles due to the increase in the scattering ability. When the QD concentration is larger than $0.31 \mathrm{wt} \%$, the scattering ability becomes saturated and the intensity begins to decrease because of the lower transmittance and light absorbance. It is evident that the composite particles can enhance the light scattering path, which may contribute to improving the QD excitation. Figure 3c shows the absorbance and PL intensity of CPF films. The CPF films exhibit an absorbance peak at about $552 \mathrm{~nm}$. The composite particle concentration affects the absorbance to a large degree, and a larger concentration leads to greater light absorbance. Subsequently, the PL intensity with an emission peak located at $567 \mathrm{~nm}$ becomes stronger as the composite particle concentration increased, and it finally reaches a balance point, which is limited by the film transmittance. The PL intensity profile reveals that more QD light could be obtained by increasing the composite particle concentration.
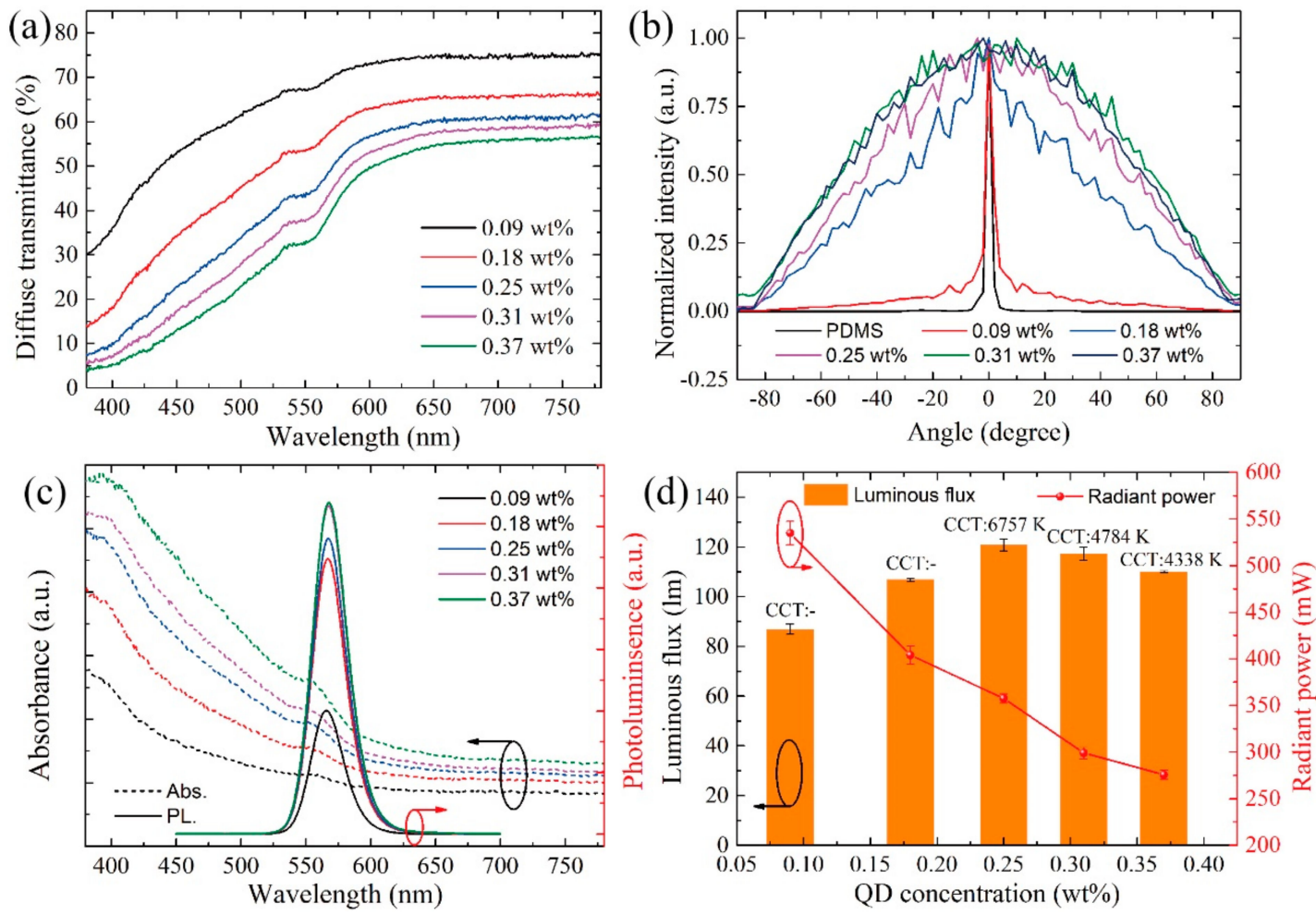

Figure 3. Optical properties of CPF films corresponding to various concentrations that varies from 0.09 to $0.37 \mathrm{wt} \%$. (a) Diffuse transmittance. (b) BTDF. (c) Absorbance and PL. (d) Luminous flux and radiant power of LED devices with CPF films. 
It is well-known that the lighting effect can be easily adjusted by controlling the phosphor concentration. CPF films with various composite particle concentrations demonstrate different lighting performances. This behavior warrants further investigation. Figure $3 \mathrm{~d}$ illustrates the optical performance of the blue COB-LED devices under excitation. By controlling the composite particle concentration, different lighting effects with various CCTs, as well as different luminous intensities, could be obtained. The luminous flux first increased and then decreased as the QD concentration increased. This is because more blue light was converted to yellow light, to which the luminous flux is most sensitive. Moreover, the light extraction could be affected by the larger concentration of the composite particles, which decreases the film transmittance. The highest luminous flux was $120.8 \mathrm{~lm}$ with a CCT of $6757 \mathrm{~K}$ when the QD concentration was $0.25 \mathrm{wt} \%$. The change in radiant power can explain the conversion, which decreases gradually as the QD concentration increased. Thus, more blue light would be absorbed by QDs and converted to QD light. Therefore, CPF films enable obtaining different lighting effects using CPF films.

To ensure the practical application potential of composite particles, a QSF structure was fabricated by direct dissolving of QD in silicone, and its performance was compared with that of the CPF structure. Both structures were adjusted the QD concentration to ensure the same lighting effect. As can be observed in Figure 4a, COB-LED-based devices with both these structures exhibit the same lighting effect with a CCT of about $4761 \mathrm{~K}$ at driving current of $150 \mathrm{~mA}$. The inset shows the structure of COB-LED device. The QD films were coated on blue COB-LED devices. Under a similar CCT, the devices with a CPF structure demonstrated better luminous efficiency (LE) than those of devices with a QSF structure. The LE for devices with a CPF structure was $73.4 \mathrm{~lm} / \mathrm{W}$, a $34.2 \%$ enhancement compared to those of devices with QSF. This can also be seen from the spectra of the two structures in Figure 4a. The intensity of the CPF structure is higher than that of QSF at both the blue and QD light wavelengths. To analyze the LE improvement clearly, the radiant power of the unexcited blue light, as well as QD light, were calculated by integrating the spectrum intensity from 380 to $500 \mathrm{~nm}$ wavelengths for blue light and 500 to $780 \mathrm{~nm}$ wavelengths for QD light; the results are illustrated in Figure 4b. For the unexcited blue light, the QSF structure exhibited a radiant power of $86.36 \mathrm{~mW}$, which was lower than that of the CPF structure $(95.52 \mathrm{~mW})$. The CPF structure contributed positively to blue light extraction, resulting in a 10.6\% enhancement of blue light radiant power. For remote QD films, a great interface loss would occur because of the total internal reflectance (TIR) between the film and air. Due to scattering from the composite particles, the TIR loss would be weakened, and the light extraction would be facilitated. The QD light radiant power of the CPF structure is $198.98 \mathrm{~mW}$, which corresponds to a $30.2 \%$ improvement than that of the QSF structure. This could be due to the spatial distribution of QDs and the utilization improvement of incident blue light. Moreover, the path length of blue light is increased due to scattering effect, thus enhancing the blue light excitation. Compared to the QSF structure, the CPF structure possesses more blue light and QD light. It can be concluded that the CPF structure contributes positively to improve the color conversion efficiency (CCE). The CCE is defined as the ratio between the emission radiant power from the QDs to the absorbed blue light radiant power, which determines the output color coordinates of LEDs. As shown in Figure $4 b$, the CCE of the CPF structure is $29.8 \%$, which is $32 \%$ higher than that of QSF; this indicates that the energy transfer of the CPF structure is more efficient than that of QSF. The CPF structure exhibits a better optical performance than the QSF structure, which proves that the proposed CPF structure is more suitable for illumination and display. 

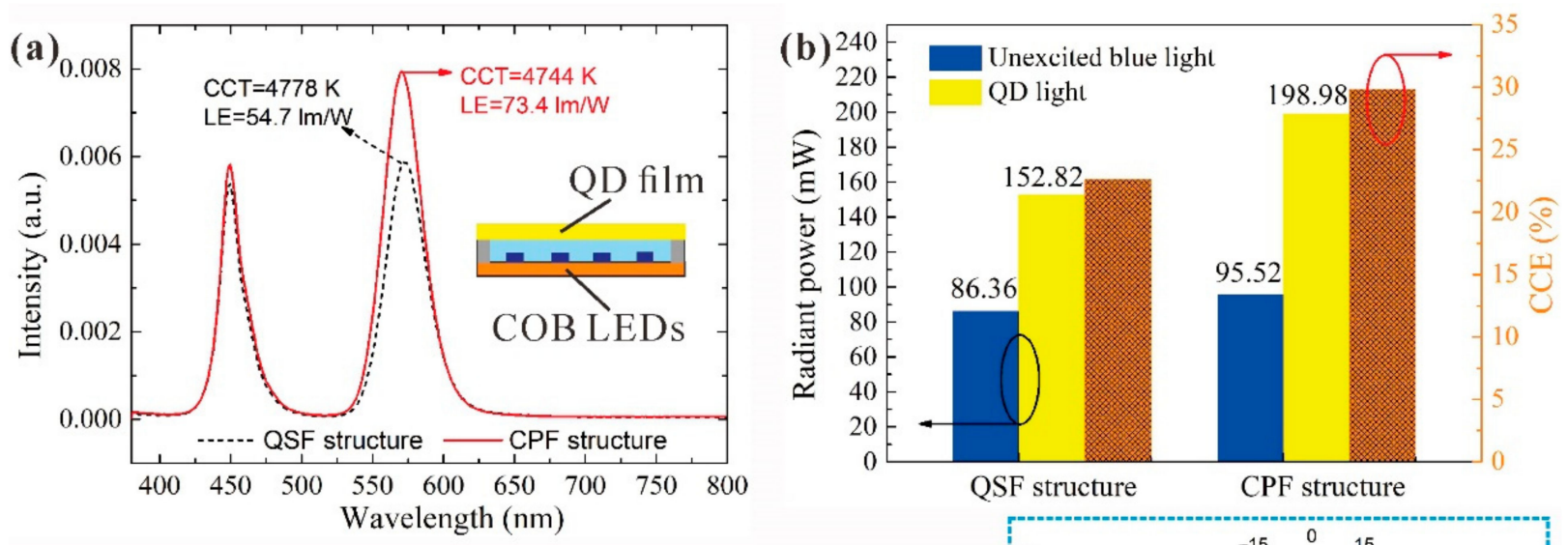

(c)
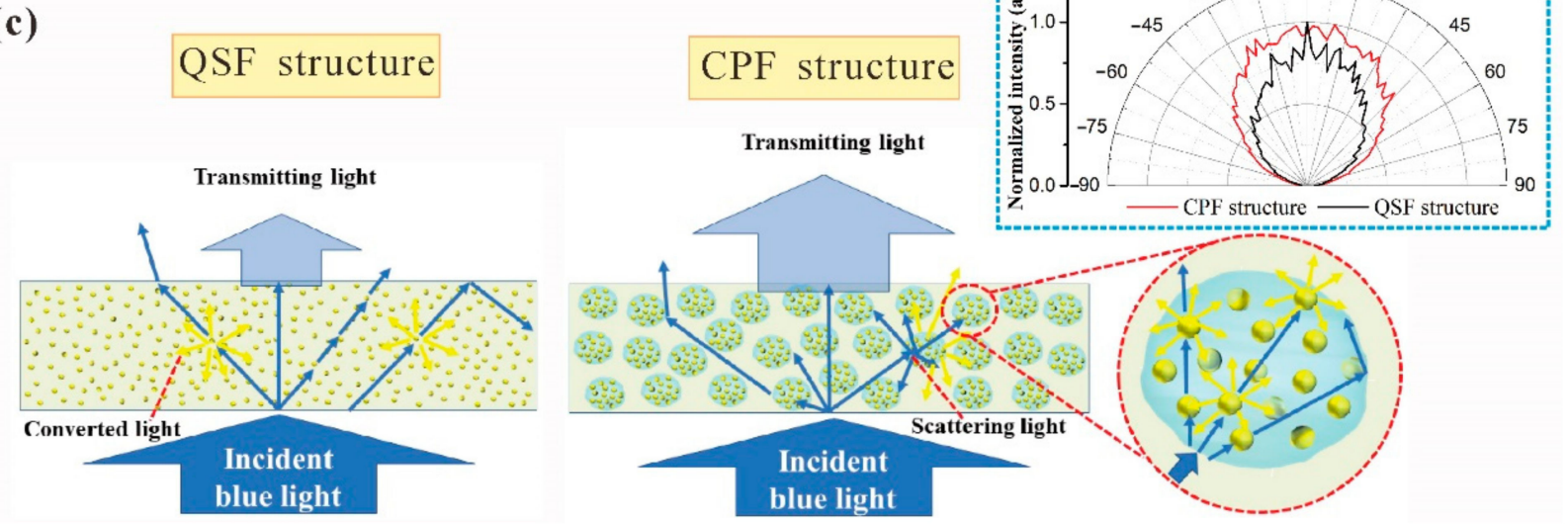

Figure 4. (a) Spectrum of devices with CPF structure and QSF structure under similar CCT at a driving current of $150 \mathrm{~mA}$. The inset is the illustration of COB-LED device structure. (b) Radiant power of blue and QD light. (c) Schematics of light conversion and extraction for different structural films. The inset is the BTDF of two types of films under the same lighting effect.

To better understand the optical enhancement, the light transfer schematic diagram is shown in Figure 4c. The different optical performances of two structure types can be explained as follows. With regard to the blue light power, due to the TIR loss between the air and silicone films, a large amount of blue light would be backscattered and re-absorbed by QD and package material. Based on the above discussion, it can be stated that the scattering ability of the CPF structure is stronger than that of the QSF structure. To further understand the scattering effect for these two kinds of structure, their BTDFs under similar lighting effects are shown in the inset. The lighting intensity of the CPF structure is more uniform than that of QSF, and the lighting angle is wider. The blue light could be scattered in different directions, thus increasing the probability of light escapes and decreasing the TIR loss. Furthermore, the scattering effect is an important feature that controls the excitation of QD photoluminescence. The blue light path length is greatly increased, which contributes to exciting more QDs. However, there exists an index mismatch between the epoxy ( 1.50) and silicone resin ( 1.41); therefore, the incident blue light is trapped in the composite. This trapping increases the blue light absorption by QDs, leading to more QD light production. Similarly, the scattering effect may also positively affect the QD light extraction. Thus, the CPF structure is beneficial to improve the light output.

To investigate the film optical stability under different driving currents varying from 50 to $1000 \mathrm{~mA}$, the luminous flux and luminous efficiency of LED devices with two kinds of structural films were studied in Figure 5a. Although the luminous flux of both structural films increases, the luminous efficiency decreases as the driving current increased. The CPF structure performs better than the QSF structure, and the improvement becomes greater as the driving current increased. At a driving current of $50 \mathrm{~mA}$, the luminous flux of the CPF 
structure is $40.3 \mathrm{~lm}$ while that of the QSF structure is $29.5 \mathrm{~lm}$, indicating an enhancement of $36.6 \%$ in the former. When the current increased to $1000 \mathrm{~mA}$, the luminous flux of the CPF structure is $567.6 \mathrm{~lm}$, which is $58.9 \%$ greater than that of the QSF structure. The luminous efficiency also demonstrates the similar tendency as the luminous flux changed. These results reveal that the CPF structure demonstrates better optical stability than the QSF structure.
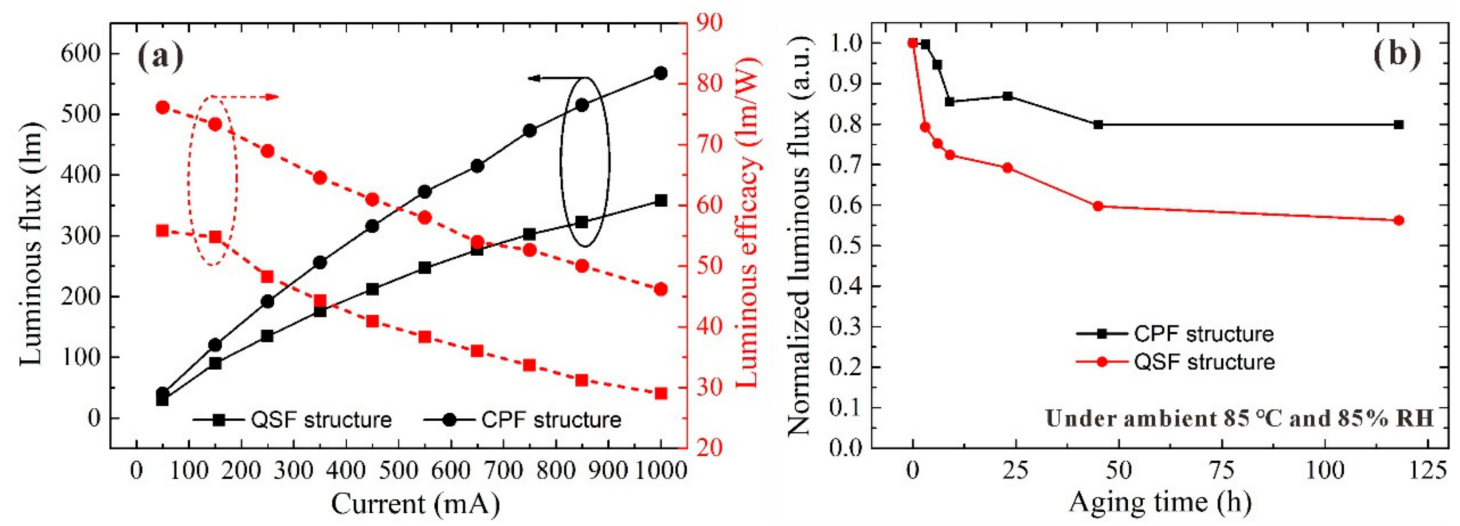

Figure 5. (a) Luminous flux and efficiency of LED devices with two kinds of structural films. (b) Normalized luminous flux during the aging process under $85^{\circ} \mathrm{C}$ and $85 \% \mathrm{RH}$ ambient.

These results demonstrate the enhancement of light efficiency of LEDs with different packaging structures. To study the film thermal and moisture stability, two types of structural films were placed in the ambient conditions of $85{ }^{\circ} \mathrm{C}$ and $85 \% \mathrm{RH}$ and with different aging times. The normalized intensity of the two types of structural films is shown in Figure 5b, which indicates the moisture effect on the photoluminescence quenching of QDs. The luminous flux decreases sharply and becomes smoother as the aging time increased, indicating that the QDs were affected by moisture severely. A comparison of the aging curves of two types of structural films reveals that the luminous flux degradation of the CPF structure is much smaller than that of QSF. After aging for $118 \mathrm{~h}$, the CPF structure could maintain a 79.8\% luminous flux, but the QSF structure exhibited a luminous flux of only $56.2 \%$. These results illustrate that the moisture stability of the QDs is significantly improved by the CPF structure. This could be attributed to the anti-moisture ability of the epoxy resin [26]. The QDs dissolved in the epoxy were well protected from the moisture, exhibiting better moisture stability than that of the QSF structure. In conclusion, the composite particles with good optical and moisture stability can be applied in the fabrication of high-performance QD-based LED devices, which can be directly used in the component package processes.

\section{Conclusions}

In summary, we fabricated a flexible, optical QD-based luminescent film with an improved moisture performance by packaging QD/epoxy composite particles (QE CPs) into silicone resin. The optical behaviors of the $\mathrm{CPF}$ films were controlled by varying the concentration of QE CPs, thus achieving different lighting effects. As the QD concentration in QE CPs increased from 0.09 to $0.37 \mathrm{wt} \%$, the diffuse transmittance of CPF films decreases gradually together with the enhancement of scattering ability. The absorptance and PL increased rapidly, which contributes to the change in radiant power and luminous flux. The radiant power decreased rapidly from 534.8 to $275.5 \mathrm{~mW}$ because of the blue light consumption, while the luminous flux increased from 86.9 to $110.0 \mathrm{~lm}$. Compared to the QSF structure under similar CCT, the CPF structure achieved a $34.2 \%$ enhancement in luminous efficiency due to the increase in light excitation and improvement in light extraction. The refractive index mismatch between the epoxy and silicone resin led to the scattering of light to improve the QD excitation and light extraction performance. The 
CCE of the CPF structure was 29.8\%, which was 32\% higher than that of a QSF structure. Furthermore, the QE CPs contributed to preventing the composite particles from water; thus, the CPF films demonstrated higher moisture stability than the QS film, exhibiting a 53.9\% improvement under $85{ }^{\circ} \mathrm{C}$ and $85 \% \mathrm{RH}$ ambient conditions after aging for $118 \mathrm{~h}$. We believe that QD/epoxy composite particles can be used for preparing flexible lightconverting films with high optical and moisture performance.

Author Contributions: Formal analysis, G.L.; J.H., Y.Y. and S.Y.; Funding acquisition, Y.T. and Z.L.; Investigation, G.L., J.H. and Y.Y.; Methodology, G.L.; Resources, Y.T., Z.L. and S.Y.; Writing-original draft, G.L.; Writing-review \& editing, J.L. and Z.L. All authors have read and agreed to the published version of the manuscript.

Funding: This research was supported by the National Natural Science Foundation of China (Nos. 51775199, 52075186 and 51735004); Project of the National and Local Joint Engineering Research Center of Semiconductor Display and Optical Communication, Zhongshan Branch (190919172214566), and Guangzhou Science and Technology Research Project (No. 202102020822).

Institutional Review Board Statement: The study did not involve humans or animals.

Informed Consent Statement: The study did not involve humans.

Data Availability Statement: Data is contained within the article.

Acknowledgments: The authors would like to acknowledge Foshan Nationstar Photoelectric Co., Ltd. Foshan, China, and good care from Simin Chen.

Conflicts of Interest: The authors declare no conflict of interest.

\section{References}

1. Xie, B.; Liu, H.; Hu, R.; Wang, C.; Hao, J.; Wang, K.; Luo, X. White-Light-Emitting Diodes: Targeting Cooling for Quantum Dots in White QDs-LEDs by Hexagonal Boron Nitride Platelets with Electrostatic Bonding (Adv. Funct. Mater. 30/2018). Adv. Funct. Mater. 2018, 28, 1871407. [CrossRef]

2. Yu, S.; Tang, Y.; Li, Z.; Chen, K.; Ding, X.; Yu, B. Enhanced optical and thermal performance of white light-emitting diodes with horizontally layered quantum dots phosphor nanocomposites. Photonics Res. 2018, 6, 90. [CrossRef]

3. Liu, W.; Zhang, Y.; Wang, C.-F.; Chen, S. Fabrication of highly fluorescent CdSe quantum dots via solvent-free microfluidic spinning microreactors. RSC Adv. 2015, 5, 107804-107810. [CrossRef]

4. Das, P.; Ganguly, S.; Banerjee, S.; Das, N.C. Graphene based emergent nanolights: A short review on the synthesis, properties and application. Res. Chem. Intermed. 2019, 45, 3823-3853. [CrossRef]

5. Ganguly, S.; Das, P.; Banerjee, S.; Das, N.C. Advancement in science and technology of carbon dot-polymer hybrid composites: A review. Funct. Compos. Struct. 2019, 1, 022001. [CrossRef]

6. Das, P.; Maruthapandi, M.; Saravanan, A.; Natan, M.; Jacobi, G.; Banin, E.; Gedanken, A. Carbon Dots for Heavy-Metal Sensing, pH-Sensitive Cargo Delivery, and Antibacterial Applications. ACS Appl. Nano Mater. 2020, 3, 11777-11790. [CrossRef]

7. Lee, H.; Kim, Y.H.; Lim, Y.W.; Jang, J.; Kang, S.M.; Bae, B.S. Flexible but mechanically robust hazy quantum dot/glass fiber reinforced film for efficiently luminescent surface light source. Adv. Opt. Mater. 2020, 8, 1902178. [CrossRef]

8. Yan, C.; Du, X.; Li, J.; Ding, X.; Li, Z.; Tang, Y. Effect of Excitation Wavelength on Optical Performances of Quantum-Dot-Converted Light-Emitting Diode. Nanomaterials 2019, 9, 1100. [CrossRef]

9. Woo, J.Y.; Kim, K.N.; Jeong, S.; Han, C.-S. Thermal behavior of a quantum dot nanocomposite as a color converting material and its application to white LED. Nanotechnology 2010, 21, 495704. [CrossRef] [PubMed]

10. Li, J.S.; Tang, Y.; Li, Z.T.; Li, J.X.; Ding, X.R.; Yu, B.H.; Yu, S.D.; Ou, J.Z.; Kuo, H.C. Toward 200 lumens per watt of quantum-dot white-light-emitting diodes by reducing reabsorption loss. ACS Nano 2021, 15, 550-562. [CrossRef]

11. Song, H.; Lee, S. Photoluminescent (CdSe)Zns quantum dot-polymethylmethacrylate polymer composite thin films in the visible spectral range. Nanotechnology 2007, 18, 55402-55406. [CrossRef]

12. Li, J.; Tang, Y.; Li, Z.; Ding, X.; Rao, L.; Yu, B. Investigation of stability and optical performance of quantum-dot-based leds with methyl-terminated-pdms-based liquid-type packaging structure. Opt. Lett. 2019, 44, 90-93. [CrossRef]

13. Kim, H.Y.; Yoon, D.-E.; Jang, J.; Lee, D.; Choi, G.-M.; Chang, J.H.; Lee, J.Y.; Lee, D.C.; Bae, B.-S. Quantum Dot/Siloxane Composite Film Exceptionally Stable against Oxidation under Heat and Moisture. J. Am. Chem. Soc. 2016, 138, 16478-16485. [CrossRef]

14. Xie, B.; Chen, W.; Hao, J.; Wu, D.; Yu, X.; Chen, Y.; Hu, R.; Wang, K.; Luo, X. Structural optimization for remote white light-emitting diodes with quantum dots and phosphor: Packaging sequence matters. Opt. Express 2016, 24, A1560-A1570. [CrossRef] [PubMed]

15. Milla, M.J.; Ulloa, J.M.; Guzmán, Á. Strong Influence of the Humidity on the Electrical Properties of InGaAs Surface Quantum Dots. ACS Appl. Mater. Interfaces 2014, 6, 6191-6195. [CrossRef] [PubMed] 
16. Martín-García, B.; Bi, Y.; Prato, M.; Spirito, D.; Krahne, R.; Konstantatos, G.; Moreels, I. Reduction of moisture sensitivity of PbS quantum dot solar cells by incorporation of reduced graphene oxide. Sol. Energy Mater. Sol. Cells 2018, 183, 1-7. [CrossRef]

17. Jo, J.H.; Kim, M.S.; Han, C.Y.; Jang, E.P.; Do, Y.R.; Yang, H. Effective surface passivation of multi-shelled inp quantum dots through a simple complexing with titanium species. Appl. Surf. Sci. 2017, 428, 906-911. [CrossRef]

18. Kwon, Y.-T.; Choi, Y.-M.; Kim, K.-H.; Lee, C.-G.; Lee, K.-J.; Kim, B.-S.; Choa, Y.-H. Synthesis of CdSe/ZnSe quantum dots passivated with a polymer for oxidation prevention. Surf. Coatings Technol. 2014, 259, 83-86. [CrossRef]

19. Jang, J.; Kim, S.; Lee, K.J. Fabrication of CdS/PMMA core/shell nanoparticles by dispersion mediated interfacial polymerization. Chem. Commun. 2007, 26, 2689-2691. [CrossRef]

20. Xuan, T.; Huang, J.; Liu, H.; Lou, S.; Cao, L.; Gan, W.; Liu, R.-S.; Wang, J. Super-Hydrophobic Cesium Lead Halide Perovskite Quantum Dot-Polymer Composites with High Stability and Luminescent Efficiency for Wide Color Gamut White Light-Emitting Diodes. Chem. Mater. 2019, 31, 1042-1047. [CrossRef]

21. Dirin, D.; Protesescu, L.; Trummer, D.; Kochetygov, I.; Yakunin, S.; Krumeich, F.; Stadie, N.; Kovalenko, M.V. Harnessing Defect-Tolerance at the Nanoscale: Highly Luminescent Lead Halide Perovskite Nanocrystals in Mesoporous Silica Matrixes. Nano Lett. 2016, 16, 5866-5874. [CrossRef] [PubMed]

22. Li, J.; Tang, Y.; Li, Z.; Ding, X.; Yu, B.; Lin, L. Largely enhancing luminous efficacy, color-conversion efficiency, and stability for quantum-dot white leds using the two-dimensional hexagonal pore structure of sba-15 mesoporous particles. ACS Appl. Mater. Interfaces 2019, 11, 18808-18816. [CrossRef] [PubMed]

23. Yu, X.; Pei, N.; Zhou, S.; Zhang, X.; Luo, X. Enhancing light efficiency and moisture stability of the quantum dots-light-emitting diodes by coating superhydrophobic nanosilica particles. IEEE Trans. Electron. Devices 2019, 66, 5196-5201. [CrossRef]

24. Lin, C.C.; Jiang, D.-H.; Kuo, C.-C.; Cho, C.-J.; Tsai, Y.-H.; Satoh, T.; Su, C. Water-Resistant Efficient Stretchable PerovskiteEmbedded Fiber Membranes for Light-Emitting Diodes. ACS Appl. Mater. Interfaces 2018, 10, 2210-2215. [CrossRef]

25. Xie, A.; Cui, T.; Cheng, R.; Wu, X.; Guo, J.; Lu, X.; Zhu, L.; Chen, S. Robust Nanofiber Films Prepared by Electro-Microfluidic Spinning for Flexible Highly Stable Quantum-Dot Displays. Adv. Electron. Mater. 2021, 7. [CrossRef]

26. Huang, J.-C.; Chu, Y.-P.; Wei, M.; Deanin, R.D. Comparison of epoxy resins for applications in light-emitting diodes. Adv. Polym. Technol. 2004, 23, 298-306. [CrossRef]

27. Chen, Z.; Liu, Z.; Shen, G.; Wen, R.; Lv, J.; Huo, J.; Yu, Y. Effect of Chain Flexibility of Epoxy Encapsulants on the Performance and Reliability of Light-Emitting Diodes. Ind. Eng. Chem. Res. 2016, 55, 7635-7645. [CrossRef]

28. Kausar, A. Epoxy and quantum dots-based nanocomposites: Achievements and applications. Mater. Res. Innov. 2019, 24, 235-243. [CrossRef]

29. Liang, G.; Yu, S.; Tang, Y.; Lu, Z.; Yuan, Y.; Li, Z.; Li, J. Enhancing luminous efficiency of quantum dot-based chip-on-board light-emitting diodes using polystyrene fiber mats. IEEE Trans. Electron. Devices 2020, 67, 4530-4533. [CrossRef]

30. Yu, S.; Fritz, B.; Johnsen, S.; Busko, D.; Richards, B.; Hippler, M.; Wiegand, G.; Tang, Y.; Li, Z.; Lemmer, U.; et al. Enhanced Photoluminescence in Quantum Dots-Porous Polymer Hybrid Films Fabricated by Microcellular Foaming. Adv. Opt. Mater. 2019, 7, 1900223. [CrossRef]

31. Mahpeykar, S.M.; Zhao, Y.; Li, X.; Yang, Z.; Xu, Q.; Lu, Z.-H.; Sargent, E.H.; Wang, X. Cellulose Nanocrystal:Polymer Hybrid Optical Diffusers for Index-Matching-Free Light Management in Optoelectronic Devices. Adv. Opt. Mater. 2017, 5, 1700430. [CrossRef]

32. Tang, Y.; Liang, G.; Chen, J.; Yu, S.; Li, Z.; Rao, L.; Yu, B. Highly reflective nanofiber films based on electrospinning and their application on color uniformity and luminous efficacy improvement of white light-emitting diodes. Opt. Express 2017, 25, 20598-20611. [CrossRef] [PubMed]

33. Kim, Y.-M.; Kostanski, L.; MacGregor, J.F. Photopolymerization of 3,4-epoxycyclohexylmethyl-3' $4^{\prime}$-epoxycyclohexane carboxylate and tri (ethylene glycol) methyl vinyl ether. Polymers 2003, 44, 5103-5109. [CrossRef] 